\title{
La Matematización y su influencia en el aprendizaje de la Matemática
}

\section{The Mathematization and its influence in Mathematics learning.}

Mercedes Leticia Lara Freire ${ }^{1}$, Marco Antonio Lara Freire ${ }^{2}$, Mayra Alejandra Pacheco Cunduri $^{3}$, Sandra Gabriela Barrazueta Rojas ${ }^{4}$.

Recibido: 10-06-2019 / Revisado: 15-07-2019 /Aceptado: 14-08-2019/ Publicado: 06-09-2019

\section{Abstract. $\quad$ DOI: https://doi.org/10.33262/cienciadigital.v3i3.3.795}

This study is based on the Vygotsky and Ausubel constructivist theory for a functional and significative learning. The theoretical framework is focused on the independent variable, the Mathematization process and the dependent variable related to Math learning. The is a field and documental research, of exploratory and correlational type. The scientific, inductive, deductive, and analytical-synthetic methods were applied. The applied technique was a survey with the Likert scale and the instrument was a structured questionnaire applied to Third Semester students of the Mechanical Industry Technology Career in the "Carlos Cisneros" Technological Institute. The main objective was to determine the processes involved in Mathematization and to detect the difficulties that this process presented; as well as, to gather both teachers and students' experiences. Once the data was collected, the charts and statistic graphs were elaborated in order to analyze and interpret the results. It was concluded that the Math teachers give more emphasis to Mathematization only to strengthen the teaching-

${ }^{1}$ Escuela Superior Politécnica de Chimborazo, Facultad de Informática y Electrónica. Riobamba, Ecuador. leticia.lara@espoch.edu.ec

${ }^{2}$ Unidad Educativa Carlos Cisneros, Área de Matemáticas. Riobamba, Ecuador. antonio.lara@educacion.gob.ec

${ }^{3}$ Escuela Superior Politécnica de Chimborazo, Facultad de Informática y Electrónica. Riobamba, Ecuador. mayra.pacheco@espoch.edu.ec

${ }^{4}$ Escuela Superior Politécnica de Chimborazo, Facultad de Informática y Electrónica. Riobamba, Ecuador. sbarrazueta@espoch.edu.ec 
learning process. Then, it was recommended that teachers encourage the Mathematization processes and relation with the environment since early ages.

Keywords: Mathematization, Significative Learning, Mathematics, Comprehension, Formalization.

\section{Resumen.}

El presente estudio se sustenta en la teoría constructivista de Vygotsky y Ausubel para conseguir un aprendizaje significativo y funcional. El aspecto teórico se centra en la variable independiente que corresponde el proceso de Matematización y la variable dependiente relacionada al Aprendizaje de Matemática. El diseño de la investigación es documental y de campo, de tipo exploratorio y correlacional, utilizando los métodos: científico, inductivo, deductivo y analítico-sintético. La técnica aplicada fue la encuesta tipo Likert en base a un cuestionario estructurado dirigido a los estudiantes del Tercer Semestre de Tecnología en Mecánica Industrial del Instituto Tecnológico Superior "Carlos Cisneros", con la finalidad de determinar los procesos involucrados en la Matematización y detectar las dificultades que encierran dichos procesos; así como, determinar las experiencias de los docentes y los estudiantes durante el proceso de enseñanza-aprendizaje. Con estos datos se procedió a la elaboración de cuadros y gráficos estadísticos, para realizar el análisis e interpretación de resultados. Se concluyó que los docentes de Matemática dan más énfasis a la utilización de la Matematización para potenciar el proceso de enseñanza- aprendizaje y se recomienda que los docentes fomenten procesos de Matematización y de relación de la matemática con el entorno desde edades tempranas.

Palabras claves: Matematización, Aprendizaje Significativo, Matemáticas, Comprensión, Formalización.

\section{Introducción.}

Las personas adquieren muchos conocimientos, habilidades, actitudes y comportamientos a través del razonamiento inductivo. Este proceso incluye las etapas de observación, definición de patrones, generalización y generación de suposiciones (Marquéz \& Morán, 2011). El razonamiento inductivo es muy importante en el aprendizaje de las matemáticas y constituye 
la base del pensamiento espacial. Por lo tanto, las actividades educativas y que se utilizarán en la educación matemática deben diseñarse de tal manera que permitan a los estudiantes generar razonamiento (Giraldo \& Ruíz, 2014). La explicación en el ámbito científico se relaciona con la mayor comprensión del por qué ocurren determinados fenómenos. Para Galileo, tal capacidad de descripción de los fenómenos se encuentra en la matemática. Según él y sus contemporáneos, la matemática ofrece la técnica para ordenar y comprender la naturaleza (Rodríguez, 2011).

Para mejorar la comprensión de los conceptos matemáticos, el aprendizaje debe comenzar con los objetos reales en la vida diaria, las matemáticas son una actividad humana y, por lo tanto, los objetos matemáticos en el aprendizaje de las matemáticas deberían comenzar con problemas reales en la vida cotidiana o con personas cercanas a la mente del estudiante. En el aprendizaje de las matemáticas se enfatiza la comprensión de hechos, conceptos, principios y operaciones; la comprensión de los conceptos matemáticos es el resultado de la construcción o reconstrucción de objetos matemáticos (Arteaga \& Macías, 2016).

El proceso de Matematización se refiere a las diversas formas de organizar actividades para mostrar características de las matemáticas como: generalidad, certeza, exactitud y brevedad. Freudenthal (1991) hace una distinción entre matematización horizontal y vertical. En la matematización horizontal, los estudiantes utilizan herramientas matemáticas para organizar y resolver un conjunto de problemas en una situación realista. La matematización vertical es el proceso de reorganización dentro de la disciplina matemática. Por lo tanto, la matematización horizontal implica pasar del mundo de la vida al mundo de los símbolos, mientras que la matemática vertical significa moverse dentro del mundo de los símbolos (Leal, 2017).

Es importante comprender varios enfoques para el aprendizaje de las matemáticas y de esa manera desarrollar las habilidades de los estudiantes, especialmente la capacidad de representación matemática. Uno de los enfoques matemáticos que proporciona un amplio espacio para que los estudiantes construyan y descubran conceptos matemáticos es la educación matemática realista. En la versión indonesia, conocida como Pendidikan Matematika Realistik (PMR). El aprendizaje realista es un enfoque de aprendizaje que coloca a las matemáticas como una actividad humana derivada del mundo real o el mundo que está 
cerca del estudiante y que los estudiantes pueden imaginar (Sembiring, 2010). En este caso, los estudiantes tienen la oportunidad activa de reinventar un concepto matemático a través de la orientación del profesor. El proceso de actividad de los estudiantes consiste principalmente en construir un modelo o concepto matemático. En RME, el proceso de matematización consiste en matematización horizontal y vertical. Lo interesante del proceso de matematización es que los estudiantes no deben ser considerados como receptores pasivos de matemáticas preparadas, sino más bien como individuos activos que pueden desarrollar su propio potencial matemático.

En el proceso de matematización, los estudiantes deben construir o desarrollar un modelo matemático de un modelo que se haya formado previamente con el conocimiento que posee (Huincaue \& Mena-Lorca, 2018). El aprendizaje de las matemáticas es como un proceso de mejora y desarrollo de ideas matemáticas. El proceso de matematización progresiva ocurre gradualmente cuando el proceso de matematización vertical forma un proceso de formalización de conceptos matemáticos de modelos matemáticos derivados de matematización horizontal. Sobre la base de los problemas que se han expuesto anteriormente, el objetivo de este estudio fue determinar las diferencias en la mejora de la capacidad de representación matemática basada en la matemática progresiva con educación matemática realista (PMR-MP) con enfoque de aprendizaje convencional (Rodríguez E. , 2013)

Las matemáticas deberían estar enmarcadas en términos aritméticos y la aritmética es universalmente aplicable porque se refiere no a objetos, sino a conceptos. Por lo tanto, el proceso de matematización está relacionado con la limpieza y con la aclaración lógica de las relaciones entre estos objetos: la matematización es de naturaleza fundamental (Velilla, 2018). El enfoque fundacional está bien arraigado en la filosofía analítica de las matemáticas. Sin embargo, se afirma que existe otro tipo de matematización que pone la aplicabilidad, en un marco dinámico y que es más relevante para el problema epistemológico de la aplicabilidad. La matemática, incluida la matemática de las matemáticas en sí, consiste en desarrollar un discurso formal que explore el universo de actividades (potenciales) y aplicaciones posibles, en lugar de objetos dados (Planas \& Arnal, 2018). 
La matemática no solo se realiza cuando los futuros profesores de matemáticas crean modelos o representaciones matemáticas de un problema con contextos de la vida real, sino también cuando el proceso de resolver estos problemas e interpretarlos en contextos de la vida real (Villa, 2015). La matemática, además de ser una actividad de los matemáticos, también es una actividad de los futuros profesores de matemáticas para comprender las situaciones cotidianas utilizando un enfoque matemático (Zambrano, 2015). De esta forma, explicar no es el simple hecho de describir con palabras las relaciones representadas en una ecuación; la explicación se da necesariamente cuando hay comprensión de las representaciones del fenómeno, tanto en el campo de las funciones que relacionan las magnitudes involucradas, como en el campo de los porqués de la existencia de tales funciones (Vizcaino \& Terrazzan, 2015).

En contextos locales los estudiantes no son capaces en su gran mayoría de aplicar los conocimientos teóricos, e inclusive las fórmulas estudiadas previamente cuando se trata de resolver problemas relacionados con la realidad o con el entorno, es decir, no han desarrollado adecuadamente el proceso de Matematización (Pérez \& Ramírez, 2011).

El presente trabajo busca establecer de qué manera influye la Matematización en el aprendizaje de la Matemática de los estudiantes del Tercer Semestre de Tecnología en Mecánica Industrial del Instituto Tecnológico Superior “Carlos Cisneros”, durante el periodo lectivo 2012-2013, con el fin de mejorar estos procesos dentro del ámbito de la formación matemática.

\section{Metodología.}

Para la realización de esta investigación se empleó el método científico, puesto que es un proceso racional y lógico sistemático, el mismo que parte de la definición y delimitación del problema, precisando objetivos claros, concretos, recolectando información confiable y pertinente; así como también, organizando, analizando e interpretando la información en base a los resultados de la observación. Este método permite presentar el conocimiento científico logrado. La investigación fue de tipo documental y de campo, no experimental. 
La población de estudio estuvo constituida por 46 estudiantes del ciclo Tecnológico del Instituto Tecnológico Superior "Carlos Cisneros", los cuales cursaban la especialización de Mecánica industrial, con un promedio de 20 años. La muestra fue de tipo aleatorio simple. Se realizaron observaciones planificadas mediante fichas para recoger y resumir los hechos observados.

Se aplicaron cuestionarios a los estudiantes y se analizaron e interpretaron los resultados. Para la elaboración del módulo se generaron fichas mnemotécnicas de bibliografía referente al tema y se realizó una recopilación de material gracias a la colaboración de los docentes del área de Matemáticas, además se aplicaron entrevistas mediante un cuestionario estructurado.

\section{Resultados}

Con la finalidad de determinar la influencia de la incidencia de la matematización en el aprendizaje de la matemática, a continuación, se tabulan los datos obtenidos en la encuesta aplicada a los 46 estudiantes del Ciclo Tecnológico del Instituto Tecnológico Superior "Carlos Cisneros."

Tabla 1. ¿El problema que plantea el maestro tiene la forma de enunciado teórico?

\begin{tabular}{lcc}
\hline \multicolumn{1}{c}{ Preguntas } & Estudiantes & Porcentaje \\
\hline Siempre & 11 & $20 \%$ \\
\hline A veces & 26 & \\
\hline Nunca & $96 \%$ \\
& & $24 \%$ \\
\hline Total & 46 & $100 \%$ \\
\hline
\end{tabular}

Fuente: Encuesta aplicada a los estudiantes

Análisis: El 80\% de los estudiantes reconoce que el maestro plantea el problema en forma de enunciado y el $20 \%$ reconoce que el maestro no lo plantea en forma de enunciado. La mayor parte de estudiantes están de acuerdo que al momento de plantear un problema matemático el profesor lo hace en forma de enunciado teórico. 
Tabla 2. ¿Cuándo un problema de Matemática se plantea en forma de enunciado, utiliza el maestro algún proceso para su resolución?

Preguntas

Siempre

$A$ veces

Nunca

Total
Estudiantes

17

14

13

46
Porcentaje

$39 \%$

$32 \%$

$29 \%$

$100 \%$

Fuente: Encuesta aplicada a los estudiantes

Análisis: El $71 \%$ de los estudiantes reconoce que el maestro que plantea el problema en forma de enunciado si utiliza un proceso para su resolución y el 29\% reconoce que el maestro no utiliza un proceso para su resolución. La mayor parte de estudiantes están de acuerdo que al momento de plantear un problema matemático en forma de enunciado teórico el profesor si utiliza un proceso para su resolución.

Tabla 3. ¿Cuándo el problema se presenta en forma de enunciado conoce y aplica usted algún proceso para resolverlo?

\begin{tabular}{lcc}
\hline \multicolumn{1}{c}{ Preguntas } & Estudiantes & Porcentaje \\
\hline Siempre & 12 & $26 \%$ \\
\hline A veces & 21 & $46 \%$ \\
\hline Nunca & 13 & $28 \%$ \\
\hline Total & 46 & $100 \%$ \\
\hline
\end{tabular}

Fuente: Encuesta aplicada a los estudiantes

Análisis: El 72\% de los estudiantes reconoce que el problema que se presenta en forma de enunciado si se conoce y se aplica un proceso para resolverlo y el $28 \%$ reconoce que el problema que se presenta en forma de enunciado se desconoce y no se puede aplicar un proceso para resolverlo. La mayor parte de estudiantes están de acuerdo que el problema que se presenta en forma de enunciado es conocido y que son capaces de aplicar, tanto un método como un procese para resolverlo. 
Tabla 4. ¿Cuándo el maestro plantea los enunciados lo hace en forma comprensible?

\begin{tabular}{lcc}
\hline \multicolumn{1}{c}{ Preguntas } & Estudiantes & Porcentaje \\
\hline Siempre & 17 & $39 \%$ \\
\hline A veces & 14 & $32 \%$ \\
\hline Nunca & 13 & $29 \%$ \\
& & $100 \%$ \\
\hline Total & 46 & \\
\hline
\end{tabular}

Fuente: Encuesta aplicada a los estudiantes

Análisis: El 71\% de los estudiantes reconoce que el maestro cuando plantea un problema si lo hace de una manera comprensible y el $29 \%$ reconoce que el maestro cuando plantea un problema no lo hace de una manera comprensible. La mayor parte de estudiantes están de acuerdo que al momento de plantear un problema matemático en forma de enunciado teórico el profesor si lo hace de una manera comprensible.

Tabla 5. ¿Señale con qué frecuencia el maestro resuelve problemas valiéndose de enunciados teóricos?

\begin{tabular}{lcc}
\hline \multicolumn{1}{c}{ Preguntas } & Estudiantes & Porcentaje \\
\hline Siempre & 12 & $26 \%$ \\
\hline A veces & 13 & $50 \%$ \\
\hline Nunca & & $24 \%$ \\
\hline Total & 21 & $100 \%$
\end{tabular}

Fuente: Encuesta aplicada a los estudiantes

Análisis: El 76\% de los estudiantes reconoce que el maestro resuelve con mayor frecuencia problemas con enunciado teórico y el $24 \%$ reconoce que el maestro no resuelve con mayor frecuencia problemas con enunciado teórico. La mayor parte de estudiantes están de acuerdo 
en que el profesor si resuelve con mayor frecuencia problemas matemáticos con enunciado teórico.

Tabla 6. ¿Si el problema se plantea en forma teórica, puede usted expresarlo (esquematizarlo) utilizando simbología matemática?

\begin{tabular}{lcc}
\hline & Estudiantes & Porcentaje \\
\hline Siempre & 7 & $15 \%$ \\
\hline A veces & 30 & $65 \%$ \\
& & \\
\hline Nunca & 9 & $20 \%$ \\
\hline Total & 46 & $100 \%$
\end{tabular}

Fuente: Encuesta aplicada a los estudiantes

Análisis: El 80\% de los estudiantes reconoce que cuando el problema se plantea en forma teórica si pueden esquematizarlo utilizando simbología matemática y el 20\% reconoce que cuando el problema se plantea en forma teórica no pueden esquematizarlo utilizando simbología matemática. La mayor parte de estudiantes están de acuerdo que cuando el problema se plantea en forma teórica si pueden esquematizarlo utilizando simbología matemática para su resolución.

Tabla 7. ¿Usted prefiere resolver problemas con enunciado teórico?

\begin{tabular}{lcc}
\hline \multicolumn{1}{c}{ Preguntas } & Estudiantes & Porcentaje \\
\hline Siempre & 12 & $26 \%$ \\
\hline A veces & 23 & $50 \%$ \\
\hline Nunca & 11 & $24 \%$ \\
\hline Total & 46 & $100 \%$ \\
\hline
\end{tabular}

Fuente: Encuesta aplicada a los estudiantes

Análisis: El 76\% de los estudiantes reconoce que si prefieren resolver problemas con enunciado teórico y el $24 \%$ reconoce que no prefiere resolver problemas con enunciado 
teórico. La mayor parte de estudiantes están de acuerdo que si prefiere resolver problemas con enunciado teórico

Tabla 8. ¿Los problemas que plantea el profesor en forma de enunciado le permite mejorar su aprendizaje?

\begin{tabular}{lcc}
\hline \multicolumn{1}{c}{ Preguntas } & Estudiantes & Porcentaje \\
\hline Siempre & 15 & $33 \%$ \\
\hline A veces & 31 & $67 \%$ \\
\hline Nunca & 0 & $0 \%$ \\
\hline Total & 46 & $100 \%$ \\
\hline
\end{tabular}

Fuente: Encuesta aplicada a los estudiantes

Análisis: El 100\% de los estudiantes reconoce que los problemas que plantea el profesor en forma de enunciado le permiten mejorar su aprendizaje.

Tabla 9. ¿El proceso que utiliza el maestro mejora el aprendizaje de la matemática?

\begin{tabular}{lcc}
\hline \multicolumn{1}{c}{ Preguntas } & Estudiantes & Porcentaje \\
\hline Siempre & 17 & $39 \%$ \\
\hline A veces & 14 & $32 \%$ \\
& & $29 \%$ \\
\hline Nunca & 13 & $100 \%$
\end{tabular}

Fuente: Encuesta aplicada a los estudiantes

Análisis: El 71\% de los estudiantes reconoce que el proceso que utiliza el maestro si ayuda a mejorar su aprendizaje de la Matemática y el 29\% reconoce que el proceso que utiliza el maestro no ayuda a mejorar su aprendizaje. La mayor parte de estudiantes están de acuerdo con que el proceso que utiliza el maestro ayuda a mejorar su aprendizaje en la Matemática. 
Tabla 10. ¿Cuándo el maestro utiliza los enunciados teóricos, los problemas se le vuelven difíciles?

\begin{tabular}{lcc}
\hline \multicolumn{1}{c}{ Preguntas } & Estudiantes & Porcentaje \\
\hline Siempre & 10 & $22 \%$ \\
\hline A veces & 30 & $65 \%$ \\
\hline Nunca & 6 & $13 \%$ \\
\hline Total & 46 & $100 \%$ \\
\hline
\end{tabular}

Fuente: Encuesta aplicada a los estudiantes

Análisis: El 80\% de los estudiantes reconoce que cuando el maestro utiliza los enunciados teóricos, los problemas se tornan difíciles y el 20\% reconoce que cuando el maestro utiliza los enunciados teóricos, los problemas no resultan difíciles. La mayor parte de estudiantes considera que cuando el maestro utiliza enunciados teóricos, los problemas se vuelven difíciles.

\section{Discusión}

La aplicabilidad de las matemáticas como un problema filosófico escapa a cualquier marco que desee restringir la filosofía de las matemáticas a una perspectiva fundamental. La posibilidad misma de que las matemáticas avancen, en el sentido dinámico de la matematización, requiere un tipo de realismo evolutivo; es decir, una concepción filosófica según la cual el mundo real aún no está completamente determinado. El tipo exploratorio de matematización es esencial para el desarrollo de las matemáticas en sí mismo tanto como lo es para nuestra comprensión eficiente del mundo. Esta perspectiva percibe los objetos no en el sentido de algún objetivismo metafísico, sino como objetos emergentes y cambiantes de la actividad humana (Vidal, 2013). Llegando a la conclusión que la matemática pone de relieve que la justificación de una teoría se orienta hacia aplicaciones futuras. En lo que respecta a la matematización, destaca la posibilidad que tuvieron los estudiantes de traducir la realidad en términos Matemáticos. 


\section{Conclusiones}

- Actualmente los docentes de Matemática dan mayor énfasis a la utilización de la matematización para potenciar el proceso de enseñanza- aprendizaje, como lo demuestra la encuesta realizada a los estudiantes. Los estudiantes tienen aún un grado de temor frente a la resolución de problemas en los cuales se tiene que realizar un proceso de matematización.

- Los conocimientos relacionados con procesos de matematización, es decir, con asuntos referentes a resolver situaciones de su entorno, no sólo que mejoran su aprendizaje, sino que fomentan el razonamiento y transforman su conocimiento en conocimiento significativo, percepción obtenida durante la elaboración del marco teórico y la investigación.

- Cuando se elabora un módulo con procesos de matematización, se refuerzan los procesos, se van relacionando conocimientos mediante el uso de algoritmos, se buscan aplicaciones y se incrementa el interés del estudiante ya que, puede visualizar la Matemática como una herramienta para entender y analizar su entorno.

\section{Referencias Bibliográficas}

Arteaga, B., \& Macías, J. (2016). Didáctica de las Matemáticas. La Rioja: UNIR.

Giraldo, M., \& Ruíz, M. (2014). Aprendizaje significativo del pensamiento espacial . Cali: Universidad Libre.

Huincaue, J., \& Mena-Lorca, J. (2018). El conocimiento de la modelación matemática desde la reflexión en la formación de profesores de matemática. Enseñanza de las Ciencias, 99-115.

Leal, A. (2017). EcuRed. Obtenido de https://www.ecured.cu/Matematizaci\%C3\%B3n

Marquéz, S., \& Morán, J. (2011). Estrategias lúdicas para el desarollo del pensamiento lógico matemático. Tesis, Milagro Ecuador.

Pérez, Y., \& Ramírez, R. (2011). Estrategias de enseñanza de la resolución de problemas matemáticos. Revista de Investigación, 169-194. 
Planas, N., \& Arnal, A. (2018). El discurso matemático del profesor. Enseñanza de las ciencias, 45-60.

Rodríguez, E. (2013). Nociones de la Teoría Matemática Realista. REDHECS, 1-15.

Rodríguez, M. (2011). La matemática y su relación con las ciencias como recurso pedagógico. Números-Revista de Didáctica de las Matemáticas, 35-49 .

Sembiring, R. (2010). Matemática Realística. ResearchGate, 11-16.

Velilla, H. (2018). Formas de matematización de la filosofía natural: Galileo y la redefinición sociocognitiva de sus matemáticas. Estudios de Filosofía.

Vidal, J. (2013). La búsqueda de la realidad o de la verdad: una aproximación a partir de la teoría sociológica. Moebio, 95-114.

Villa, J. (2015). Modelación matemática a partir de problemas de enunciados verbales: un estudio de caso con profesores de matemáticas. Magis-Revista Internacional de Investigación en Educación, 133-148.

Vizcaino, D., \& Terrazzan, A. (2015). Diferencias trascendentales entre matematización de la física y matematización para la enseñanza de la física. Scielo, 95-111.

Zambrano, A. (2015). La práctica de la enseñanza de las matemáticas a través de las situaciones de contingencia. Barcelona: Universodad Autónoma de Barcelona.

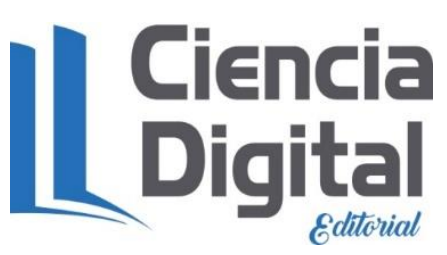




\section{PARA CITAR EL ARTÍCULO INDEXADO.}

Lara Freire, M., Lara Freire, M., Pacheco Cunduri, M., \& Barrazueta Rojas, S. (2019). La Matematización y su influencia en el aprendizaje de la Matemática. Ciencia Digital, 3(3.3), $196-206$. https://doi.org/10.33262/cienciadigital.v3i3.3.795

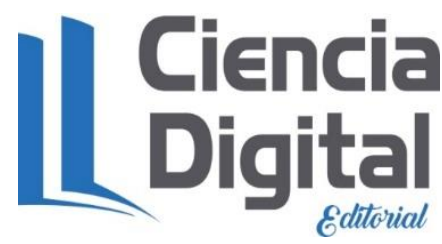

El artículo que se publica es de exclusiva responsabilidad de los autores y no necesariamente reflejan el pensamiento de la Revista Ciencia Digital.

El artículo queda en propiedad de la revista y, por tanto, su publicación parcial y/o total en otro medio tiene que ser autorizado por el director de la Revista Ciencia Digital.
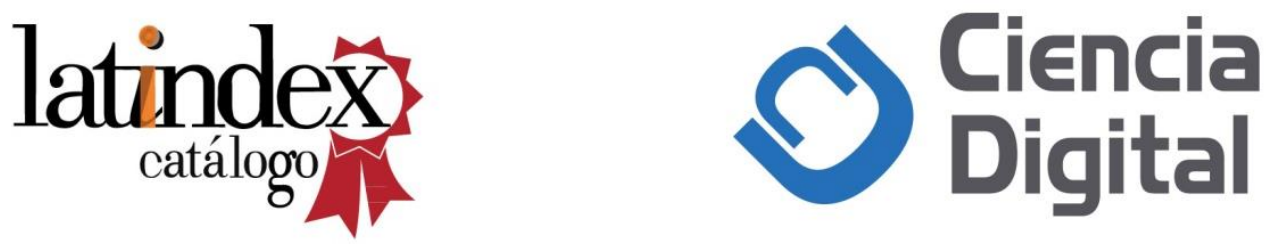